\title{
Synchronization in two-level quantum systems
}

\author{
Álvaro Parra-López $\odot$ and Joakim Bergli \\ Department of Physics, University of Oslo, NO-0316 Oslo, Norway
}

(Received 26 April 2019; accepted 6 May 2020; published 4 June 2020)

\begin{abstract}
Recently, it was shown that dissipative quantum systems with three or more levels are able to synchronize to an external signal. Some researchers have stated that this is not possible for two-level systems, as those systems lack a stable limit cycle in unperturbed dynamics, but others have demonstrated, under a different definition of synchronization, that it is possible in qubits in models which include other elements. We show how a quantum two-level system can be understood as containing a valid limit cycle as the starting point of synchronization and that it can synchronize its dynamics to an external weak signal. This is demonstrated by analytically solving the Lindblad equation of a two-level system coupled to an environment, determining the steady state. This is a mixed state with contributions from many pure states, each of which provides a valid limit cycle. We show that this is sufficient to phase lock the dynamics to a weak external signal, hence clarifying synchronization in two-level systems. We use the Husimi $Q$ representation to analyze the synchronization region, defining a synchronization measure which characterizes the strength of the phase locking. Also, we study the stability of the limit cycle and its deformation with the strength of the signal in terms of the components of the Bloch vector of the system. Finally, we generalize a model of the three-level system to illustrate how the stationary fixed point of that model can be changed into a limit cycle similar to the one that we describe for the two-level system.
\end{abstract}

DOI: 10.1103/PhysRevA.101.062104

\section{INTRODUCTION}

The phenomenon of synchronization occurs in many different situations and has been extensively studied for many years. If an autonomous oscillating system is coupled to another such system or to an external driving force, it can synchronize its frequency and phase to the external system. Examples are coupled pendulums, circadian rhythms in living systems, and synchronization of fireflies flashing. Common to these systems are the fact that they need to have a stable limit cycle, which means they must be dissipative, so that they can return to the stable cycle after a perturbation, and contain an energy source, so that they can sustain oscillations indefinitely in the presence of dissipation [1].

One well-studied example of classical synchronization is the van der Pol oscillator model [1,2]. Some years ago, the van der Pol model was reformulated in terms of a quantum system [3,4], and it was shown that when the system is far from the ground state, synchronization in quantum systems is analogous to classical synchronization of the same system in the presence of noise [1]. When we are close to the ground state, this correspondence is changed because the discreteness of the energy levels becomes important. It is therefore interesting to study synchronization in quantum systems with a small number of energy levels.

The natural idea is to synchronize a two-level system (TLS) either with another two-level system, as was done in Refs. [5,6], or with an external signal. The latter was discussed in Ref. [7], with the conclusion that it is not possible to have a stable limit cycle in the dynamics of a dissipative TLS, and therefore synchronization cannot occur. However, in Refs. [8,9], it is claimed that synchronization between a qubit and a driven external signal is possible, although there is no discussion about the limit cycle of the system or how it may occur. A brief discussion of the synchronization of a single qubit to an external signal is also present in Ref. [10]. We show how one can understand the appearance of a valid limit cycle, which is an essential starting point for synchronization, if one aims to relate the quantum version of this phenomena to its classical counterpart. In this context, the system is not completely phase locked, and therefore if we accept that the quantum system is similar to a classical system with noise, as is the case for the van der Pol oscillator [3,4] (and all quantum systems in general [11-13]), a TLS is in fact capable of synchronization, and the following considerations allow us to understand why.

Our system is in contact with an environment so that it is able to gain and emit energy, hence creating the dissipating frame synchronization requires. Solving the Lindblad equation for the system in the absence of any external signal, one finds that the stationary solutions are mixed states that are constant in time, lying on the rotation axis of the Bloch sphere, which we will take to be the $z$ axis. As was stated in Ref. [7], this is not a valid limit cycle and it seems that it cannot form the starting point for synchronization. Nevertheless, as these states are mixed, we must understand that the system is in a probability mixture of some pure states. While the ensemble of pure states which generates a given mixed state is not unique, we can choose them to be on that circle on the surface of the Bloch sphere which is in the plane normal to the $z$ axis and which has the given mixed state at the center; see Fig. 1. Each of these states is then rotating and provides a limit cycle, while the mixed state of the ensemble is stationary. This argument does not apply to the three-level system discussed in Ref. [7], since in that case the steady state in the absence of an external signal is an eigenstate of the Hamiltonian, and only 


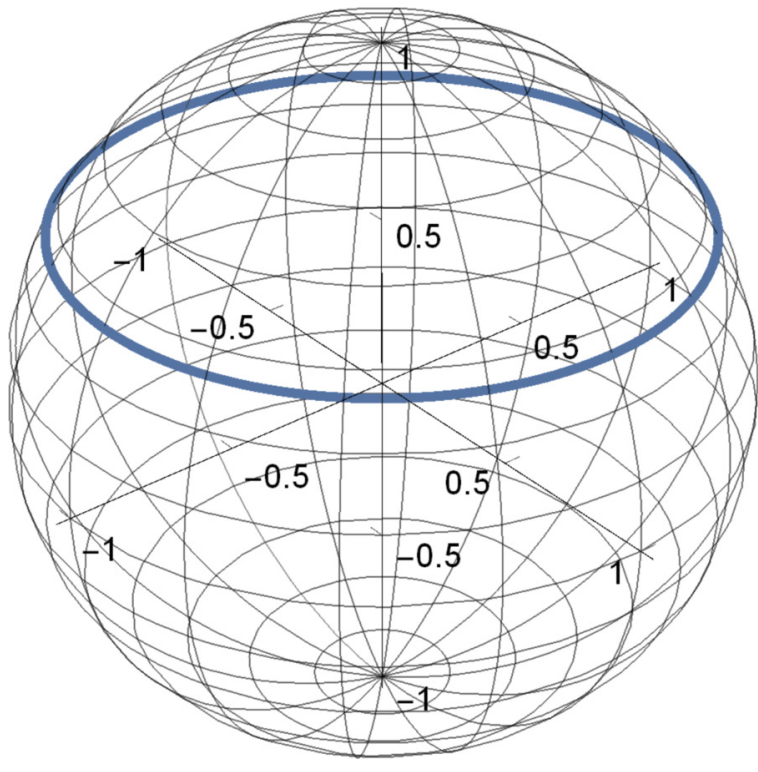

FIG. 1. We can realize the mixed steady state as a probabilistic ensemble of the states located on the surface of the Bloch sphere (state space) in the plane normal to the $z$ axis (blue circle). Each of these states move along the same circle, with a periodic motion that will correspond to a limit cycle in the phase space. In this example, we have chosen $\frac{\Gamma_{g}}{\Gamma_{d}}=3$.

when we introduce an external signal do we obtain a cycle by driving the system away from this state. We will illustrate at the end how to modify this model so that the stationary state in the absence of a signal is a limit cycle in the same sense as for the TLS we consider here. In the following, we will show that a two-level system indeed displays all the signatures of synchronization as was demonstrated for a three-level system (spin 1) [7], but also that it is possible to understand this synchronization as arising from the limit cycles provided by the mixed steady state in the unperturbed dynamics.

\section{MODEL AND LIMIT CYCLE}

We consider a two-level system characterized by the Hamiltonian

$$
\hat{H}_{0}=\frac{\hbar}{2} \omega_{0} \mathbf{n} \cdot \hat{\boldsymbol{\sigma}},
$$

where $\hat{\boldsymbol{\sigma}}$ is a vector containing the three Pauli matrices. The density operator for this system can be written as

$$
\hat{\rho}^{\prime}=\frac{1}{2}\left(\mathbb{1}+\mathbf{m}^{\prime} \cdot \hat{\boldsymbol{\sigma}}\right),
$$

where $\mathbf{m}^{\prime}$ is the Bloch vector [14]. Whenever $\mathbf{m}^{\prime}$ is not pointing in the same direction as $\mathbf{n}$, the Bloch vector precesses around the $n$ axis with frequency $\omega_{0}$, which we call the natural frequency of the system. However, although this may indicate the existence of a limit cycle, states should be stable under perturbations. Thus, we must consider a model in which the system is capable of gaining and losing energy. If we choose $\mathbf{n}$ such that it points toward the $z$ direction in the Bloch sphere, we can write the Hamiltonian as

$$
\hat{H}_{0}=\frac{\hbar}{2} \omega_{0} \hat{\sigma}_{z} .
$$

We transform to a frame rotating with the natural frequency $\omega_{0}$, defining the density matrix in the rotating frame as $\hat{\rho}=$ $\hat{T}_{\omega_{0}} \hat{\rho}^{\prime} \hat{T}_{\omega_{0}}^{\dagger}$, where

$$
\hat{T}_{\omega_{0}}=e^{i \frac{\omega_{0}}{2}} \hat{\sigma}_{z},
$$

and denote the corresponding Bloch vector $\mathbf{m}$. In this frame, the Lindblad equation including gain and damping is [14]

$$
\frac{d \hat{\rho}}{d t}=\frac{\Gamma_{g}}{2} \mathcal{D}\left[\hat{\sigma}_{+}\right] \hat{\rho}+\frac{\Gamma_{d}}{2} \mathcal{D}\left[\hat{\sigma}_{-}\right] \hat{\rho},
$$

where $\Gamma_{g}$ and $\Gamma_{d}$ are the gain and damping rates, $\mathcal{D}[\hat{\mathcal{O}}] \hat{\rho}=$ $\hat{\mathcal{O}} \hat{\rho} \hat{\mathcal{O}}^{\dagger}-\frac{1}{2}\left\{\hat{\mathcal{O}}^{\dagger} \hat{\mathcal{O}}, \hat{\rho}\right\}$ is the Lindblad superoperator, and $\hat{\sigma}_{+}$ and $\hat{\sigma}_{-}$are the ladder operators for the system, $\hat{\sigma}_{ \pm}=\frac{1}{2}\left(\hat{\sigma}_{x} \pm\right.$ $\left.i \hat{\sigma}_{y}\right)$. This equation is equivalent to the one studied in Ref. [7].

In terms of the Bloch vector components, we find the following equations:

$$
\begin{aligned}
& \dot{m}_{x}=-\frac{1}{4}\left(\Gamma_{d}+\Gamma_{g}\right) m_{x}, \\
& \dot{m}_{y}=-\frac{1}{4}\left(\Gamma_{d}+\Gamma_{g}\right) m_{y}, \\
& \dot{m}_{z}=\frac{1}{2}\left[\Gamma_{g}\left(1-m_{z}\right)-\Gamma_{d}\left(1+m_{z}\right)\right] .
\end{aligned}
$$

As we are working in a frame rotating with the natural frequency of the system, a point that precesses in the nonrotating frame should be now a fixed point. Thus, we look for stationary solutions, i.e., $\hat{\rho}=0, \dot{\mathbf{m}}=0$. The solution is then

$$
m_{x}=0 ; \quad m_{y}=0 ; \quad m_{z}=\frac{\Gamma_{g}-\Gamma_{d}}{\Gamma_{g}+\Gamma_{d}} .
$$

For the ground state $|\downarrow\rangle$ and the excited state $|\uparrow\rangle$ which correspond to the Bloch vectors $\mathbf{m}_{g}=(0,0,-1)$ and $\mathbf{m}_{e}=$ $(0,0,1)$, respectively, we do not expect a limit cycle, as the state is a fixed point. However, even if the solution for any of the other cases is lying on the $z$ axis, we must remember that they are mixed states. This means that our solution is a mixture of pure states, each of them weighted with a certain probability. It is not a superposition and our system is for sure in any of those pure states, but only in one of them at the same time.

Thus, the limit cycle is provided given that each of those possible pure states that make up our mixed state would precess around the $z$ axis once we move back to the nonrotating frame. For example, a mixture of states lying on a circle on the surface of the Bloch sphere, in a plane normal to the $z$ axis and with the steady state in the center, as illustrated in Fig. 1. However, the stationary state can always be realized as a mixture of the pure states $|\downarrow\rangle$ and $|\uparrow\rangle$, which are not precessing, and this means that the above argument is not fully convincing. Having this picture in mind, we will now demonstrate that a TLS indeed allows synchronization in the presence of an external signal.

\section{SYNCHRONIZATION OF THE TLS}

In order to synchronize our system with an external signal, we use a classical drive [7] of frequency $\omega$ and strength $\epsilon$. In the rotating-wave approximation [14], it is given by the Hamiltonian

$$
\hat{H}_{\text {signal }}=i \hbar \frac{\epsilon}{4}\left(e^{i \omega t} \hat{\sigma}_{-}-e^{-i \omega t} \hat{\sigma}_{+}\right) .
$$


If we want to move to a frame rotating with the frequency of the signal, the transformation operator we must apply to our Lindblad equation is

$$
\hat{T}_{\omega}=e^{i \frac{\omega}{2} \hat{\sigma}_{z} t}
$$

giving

$$
\frac{d \hat{\rho}}{d t}=-\frac{i}{2}\left[\Delta \hat{\sigma}_{z}+\epsilon \hat{\sigma}_{y}, \hat{\rho}\right]+\frac{\Gamma_{g}}{2} \mathcal{D}\left[\hat{\sigma}_{+}\right] \hat{\rho}+\frac{\Gamma_{d}}{2} \mathcal{D}\left[\hat{\sigma}_{-}\right] \hat{\rho},
$$

where $\Delta=\omega_{0}-\omega$.

Again, we obtain the evolution equations for the Bloch vector components,

$$
\begin{aligned}
& \dot{m}_{x}=-\frac{1}{4}\left(\Gamma_{d}+\Gamma_{g}\right) m_{x}-\Delta m_{y}+\epsilon m_{z}, \\
& \dot{m}_{y}=\Delta m_{x}-\frac{1}{4}\left(\Gamma_{d}+\Gamma_{g}\right) m_{y}, \\
& \dot{m}_{z}=\frac{1}{2}\left[\Gamma_{g}\left(1-m_{z}\right)-\Gamma_{d}\left(1+m_{z}\right)-2 \epsilon m_{x}\right],
\end{aligned}
$$

with the stationary solution

$$
\begin{aligned}
& m_{x}=\frac{4 \epsilon\left(\Gamma_{g}-\Gamma_{d}\right)}{\left(\Gamma_{d}+\Gamma_{g}\right)^{2}+8\left(\epsilon^{2}+2 \Delta^{2}\right)}, \\
& m_{y}=\frac{16 \epsilon \Delta\left(\Gamma_{g}-\Gamma_{d}\right)}{\left(\Gamma_{d}+\Gamma_{g}\right)\left[\left(\Gamma_{d}+\Gamma_{g}\right)^{2}+8\left(\epsilon^{2}+2 \Delta^{2}\right)\right]}, \\
& m_{z}=\frac{\left(\Gamma_{d}-\Gamma_{g}\right)\left[\left(\Gamma_{d}+\Gamma_{g}\right)^{2}+16 \Delta^{2}\right]}{\left(\Gamma_{d}+\Gamma_{g}\right)\left[\left(\Gamma_{d}+\Gamma_{g}\right)^{2}+8\left(\epsilon^{2}+2 \Delta^{2}\right)\right]} .
\end{aligned}
$$

In order to obtain the state operator in the nonrotating frame, we transform back with $\hat{T}_{\omega}$, and thus the state operators are related by $\hat{\rho}^{\prime}=\hat{T}_{\omega}^{\dagger} \hat{\rho} \hat{T}_{\omega}$. Therefore, the Bloch vector in the nonrotating frame will be given by

$$
\begin{aligned}
& m_{x}^{\prime}=m_{x} \cos \omega t-m_{y} \sin \omega t, \\
& m_{y}^{\prime}=m_{x} \sin \omega t+m_{y} \cos \omega t, \\
& m_{z}^{\prime}=m_{z} .
\end{aligned}
$$

When transforming back to the nonrotating frame, $m_{x}^{\prime}$ and $m_{y}^{\prime}$ will vary in time with the frequency of the signal, which means that the system phase locks to the external force.

Equations (12) give steady states with nonzero transverse Bloch vector components $m_{x}$ and $m_{y}$, provided the damping and gain rates are not equal and the strength of the signal is different than zero. Thus, Eq. (13) will give a precessing vector with frequency $\omega$ in the nonrotating reference frame. Also, it is not difficult to show that it does not matter which is the initial state: After some transient, the motion will be the one described by the steady solution.

When both $m_{x}$ and $m_{y}$ are zero, the steady state is lying on the $z$ axis and will still be a fixed point in the nonrotating frame $\left(m_{z}^{\prime}=0\right.$ if $\left.\Gamma_{g}=\Gamma_{d}\right)$. Therefore, there is no synchronization in this case.

In order to visualize the behavior of the system, we follow Ref. [7] and use the Husimi $Q$ representation adapted to spin systems [15]. This is a quasiprobability distribution that allows us to represent the phase space of the two-level system and is defined by

$$
Q(\theta, \phi)=\frac{1}{2 \pi}\langle\theta, \phi|\hat{\rho}| \theta, \phi\rangle .
$$

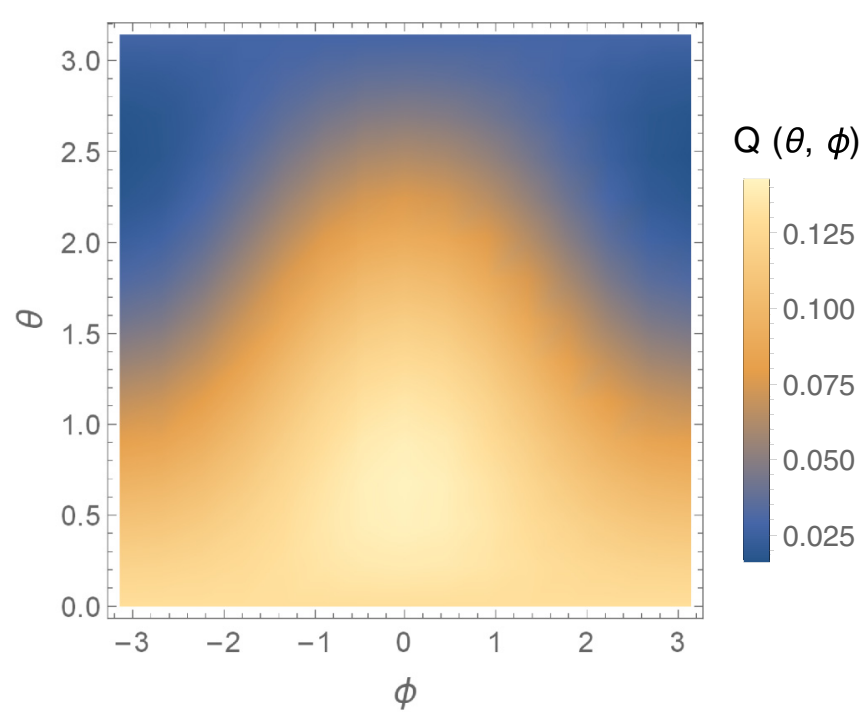

FIG. 2. $Q$ function of the steady state for $\epsilon=2 \min \left\{\Gamma_{g}, \Gamma_{d}\right\}$, $\Delta=0$, and $\frac{\Gamma_{g}}{\Gamma_{d}}=10$. The distribution is peaked around $\theta=0$ because it is the gain rate that dominates and around $\phi=0$ because we are synchronizing to a resonant signal (there is no detuning). Complete phase locking does not occur and the $Q$ function is nonzero everywhere. Note that the units of $\epsilon$ and $\Delta$ will depend on those chosen for the rates.

Here $|\theta, \phi\rangle$ are spin-coherent states, which in the case of a TLS are the eigenstates of the spin operator $\sigma_{\mathbf{n}}=\mathbf{n} \cdot \hat{\boldsymbol{\sigma}}$ along the axis given by the unit vector $\mathbf{n}$, which has polar coordinates $\theta$ and $\phi$. These are nothing but the pure states at the corresponding point on the Bloch sphere in terms of the angles $\theta$ and $\phi$. Therefore, the $Q$ representation tells us how every pure state (corresponding to a pair of angles in the Bloch sphere) that contributes to the state operator $\hat{\rho}$ is weighted:

$$
\langle\theta, \phi|\hat{\rho}| \theta, \phi\rangle=\sum_{n} P_{n}\left|\left\langle\theta, \phi \mid \psi_{n}\right\rangle\right|^{2},
$$

where $\hat{\rho}=\sum_{n} P_{n}\left|\psi_{n}\right\rangle\left\langle\psi_{n}\right|$.

Given the solution, Eq. (12), it is easy to find that the $Q$ function of the steady states, as a function of the components of the Bloch vector, is

$$
\begin{aligned}
Q(\theta, \phi)= & \frac{1}{4 \pi}\left[1+m_{x} \cos \phi \sin \theta+m_{y} \sin \phi \sin \theta\right. \\
& \left.+m_{z} \cos \theta\right] .
\end{aligned}
$$

Figure 2 shows the $Q$ function for the case where there is no detuning and the gain rate is larger than the damping rate, $\Gamma_{g}>\Gamma_{d}$. As expected, the states that contribute the most to the mixture of the steady state are those corresponding to $\theta=$ 0 . Also, the distribution is located around $\phi=0$. The system is phase locked in the sense that the state is made up mostly by contributions from a specific $\phi$ region.

On the other hand, in Fig. 3, it is the damping rate that dominates. Hence, we expect higher values of the $Q$ function at $\theta$ values close to $\pi$. When this is the case, for no detuning, the distribution is situated at $\phi=\pi$. However, in Fig. 3 the detuning shifts the phase toward $\phi=\frac{\pi}{2}$. In terms of the Bloch vector, the detuning makes $m_{y}$ nonzero, and therefore the 


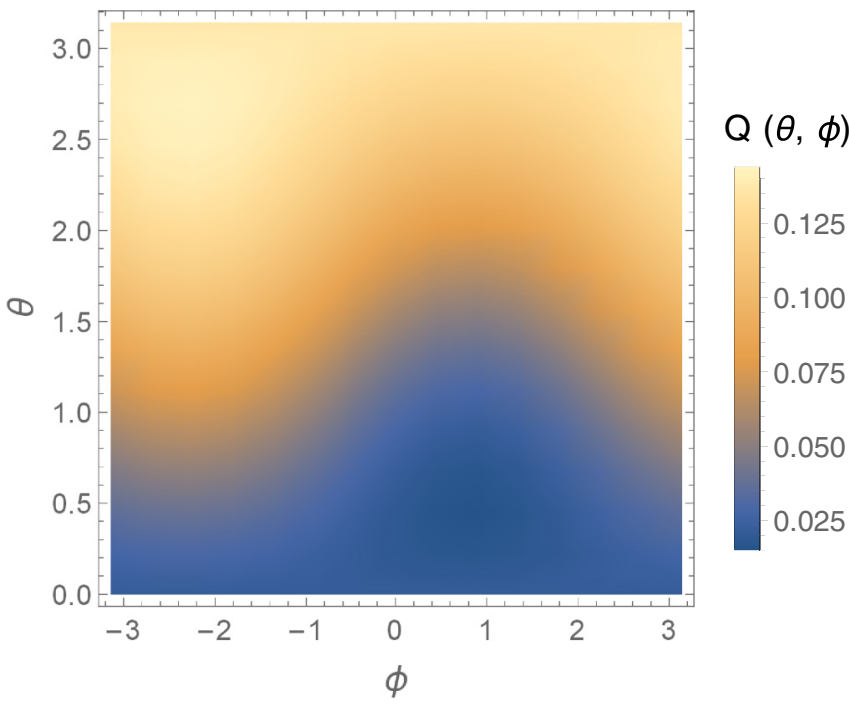

FIG. 3. $Q$ function of the steady state for $\epsilon=2 \min \left\{\Gamma_{g}, \Gamma_{d}\right\}$, $\Delta=3 \min \left\{\Gamma_{g}, \Gamma_{d}\right\}$, and $\frac{\Gamma_{g}}{\Gamma_{d}}=0.1$. Since $\Gamma_{d}>\Gamma_{g}$, we find the higher values of the function around $\theta=\pi$. Because of the detuning, the distribution is displaced along the $\phi$ axis, moving away from $\phi=\pi$, which is where it would be located if $\Delta=0$.

projection in the $x y$ plane is not a vector lying on the $x$ axis, which is the case for Fig. 2, where there is no detuning.

As a next step, we attempt to measure how strong is synchronization defining a synchronization measure.

\section{SYNCHRONIZATION REGION}

Even if we are able to observe phase locking when plotting the $Q$ function, we would like to characterize its strength. There is a tool that allows us to do it, and following the work done in Ref. [7], we define a synchronization measure,

$$
S(\phi)=\int_{0}^{\pi} d \theta \sin \theta Q(\theta, \phi)-\frac{1}{2 \pi} .
$$

This is identically zero when there is no synchronization, i.e., when only $m_{z}$ is nonzero. Explicitly performing the integral over $\theta$, we find that

$$
S(\phi)=\frac{1}{8}\left(m_{x} \cos \phi+m_{y} \sin \phi\right) .
$$

Thus, $S(\phi)$ is going to be greater as the steady state is farther from the $z$ axis.

Figures 4-6 are useful for understanding the synchronization dynamics. In Fig. 4, we can observe how the detuning drives the phase of the system toward positive or negative $\phi$ if the detuning $\left(\Delta=\omega_{0}-\omega\right)$ is positive or negative. As the absolute value of $\Delta$ increases, the synchronization weakens. Note that for this figure we have $\frac{\Gamma_{d}}{\Gamma_{g}}<1$ and we observe in-phase synchronization. If instead we use $\frac{\Gamma_{d}}{\Gamma_{g}}>1$, we would observe antiphase synchronization, which means that the maximal value of $S(\phi)$ will be at $\phi=\pi$ for $\Delta=0$.

On the other hand, Fig. 5 takes into account the effect of growing signal strength on the synchronization measure. It is clear that the synchronization is stronger for a greater $\epsilon$ (both in- and antiphase synchronization), but we must keep

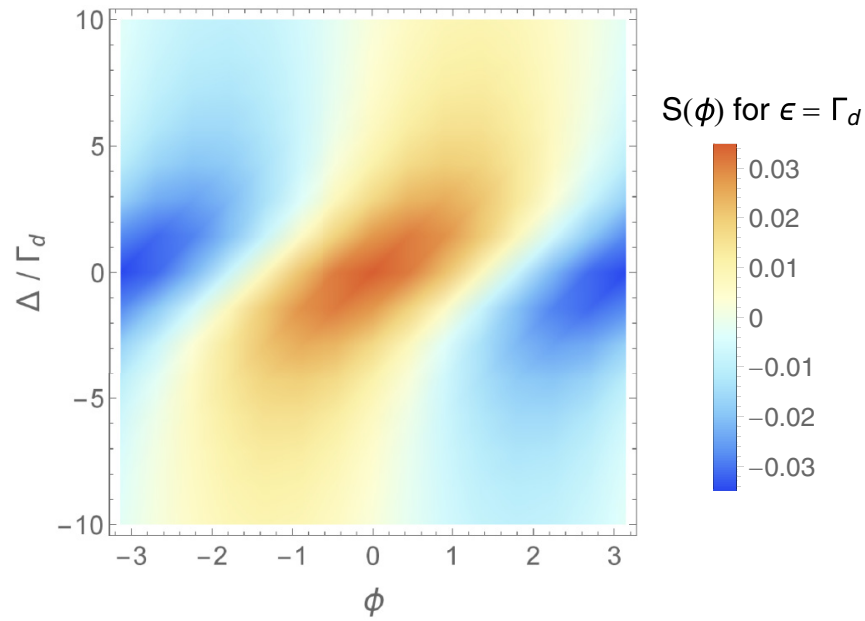

FIG. 4. $S(\phi)$ for $\epsilon=\Gamma_{d}$ for different $\Delta, \frac{\Gamma_{d}}{\Gamma_{g}}=0.1$. As expected, the phase locking is stronger when there is no detuning. When $\Delta$ is positive or negative, the maximal value of $S(\phi)$ is shifted toward $\phi=\pi$ or $\phi=-\pi$, respectively.

in mind that a very strong signal would take us out of the synchronization regime.

Finally, the Arnold tongue that is characteristic of every synchronized system is displayed in Fig. 6. The shape is that of the tongue for a spin-1 system (Fig. 3 from Ref. [7]), and it is worth mentioning that $S(\phi)$ is different from zero everywhere except for the case when $\epsilon=0$. The state will always precess with the frequency of the signal but depending on the size of $m_{x}$ and $m_{y}$, the synchronization measure will be greater or smaller, telling us the strength of the phase locking.

We have seen that the results for the two-level system are equivalent to those obtained in Ref. [7] for the three-level system. Moreover, we have explained the appearance of the limit cycle for the present case. Nevertheless, in order to stay

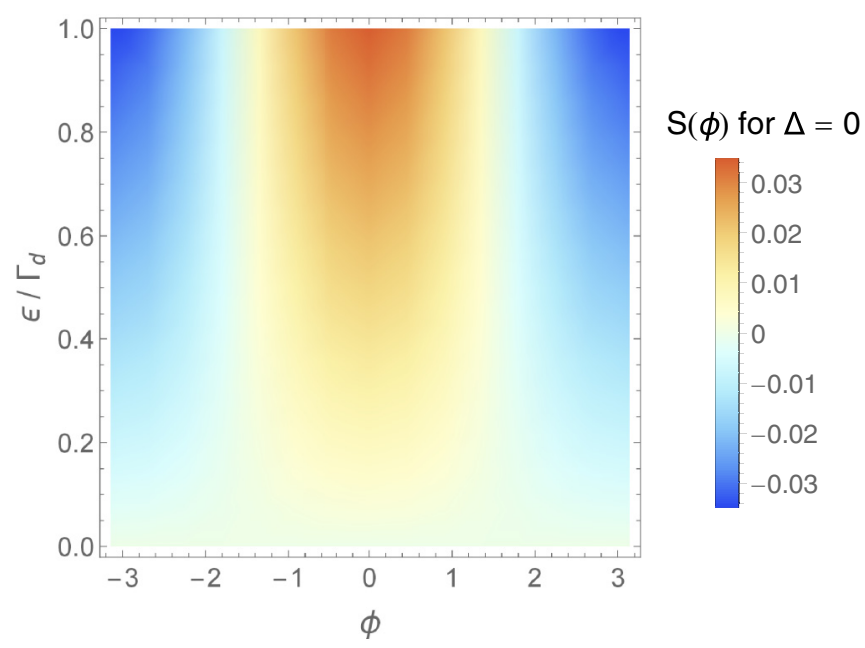

FIG. 5. $S(\phi)$ for $\Delta=0$ for different $\epsilon$. We study how the strength of the signal modifies the strength of the phase locking. We observe that greater strength of the signal is correlated with stronger phase locking. It is because there is no detuning that the highest values locate at $\phi=0$ (in-phase synchronization), as we could deduce from Fig. 4. 


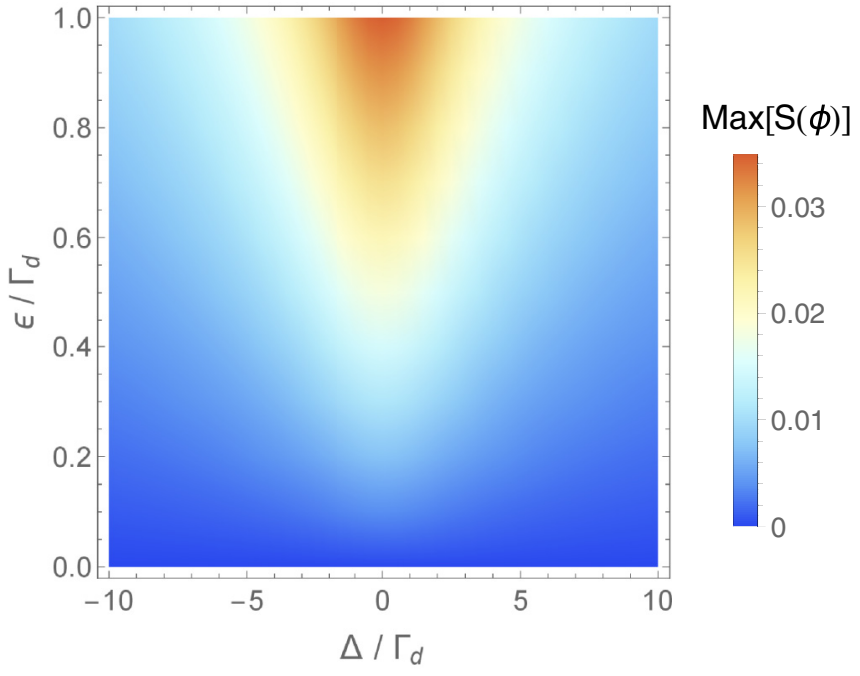

FIG. 6. Arnold tongue of the system. We plot the maximum value of $S(\phi)$ as function of the strength $\epsilon$ and the detuning $\Delta$, with $\frac{\Gamma_{d}}{\Gamma_{g}}=0.1$. This plot resembles the one in Ref. [7]. Unless $\epsilon=0$ or $\Gamma_{g}=\Gamma_{d}$, (12) will always give nonzero $m_{x}$ and $m_{y}$, and therefore $\max [S(\phi)]$ will be nonzero, even if it is so small that the synchronization is negligible.

in the synchronization regime, the strength of the external signal should not be too large, and we will analyze how the limit cycle is distorted with $\epsilon$ in the following section.

\section{STRENGTH OF THE SIGNAL AND DEFORMATION OF THE LIMIT CYCLE}

Recall the solution we found for the Bloch vector of the steady state of the synchronized two-level system in the rotating frame, Eq. (12). For small $\epsilon$, we can expand each of the Bloch vector components in powers of $\epsilon$ :

$$
\begin{aligned}
& m_{x} \approx A \cdot \epsilon\left[1-K \epsilon^{2}\right] \\
& m_{y} \approx B \epsilon\left[1-K \epsilon^{2}\right], \\
& m_{z} \approx C\left[1-K \epsilon^{2}\right],
\end{aligned}
$$

where

$$
\begin{gathered}
A=\frac{4\left(\Gamma_{g}-\Gamma_{d}\right)}{\left(\Gamma_{d}+\Gamma_{g}\right)^{2}+16 \Delta^{2}}, \\
B=\frac{16 \Delta\left(\Gamma_{g}-\Gamma_{d}\right)}{\left(\Gamma_{d}+\Gamma_{g}\right)\left[\left(\Gamma_{d}+\Gamma_{g}\right)^{2}+16 \Delta^{2}\right]}, \\
C=\frac{\Gamma_{d}-\Gamma_{g}}{\Gamma_{d}+\Gamma_{g}}, \\
K=\frac{8}{\left(\Gamma_{d}+\Gamma_{g}\right)^{2}+16 \Delta^{2}} .
\end{gathered}
$$

Note that the first nonconstant term of (19c) is quadratic in $\epsilon$ while both (19a) and (19b) are linear in $\epsilon$. Thus, we expect that for small signal strengths, the Bloch vector components that change with $\epsilon$ are $m_{x}$ and $m_{y}$, showing that the system phase locks to the external signal. $m_{z}$ will remain approximately constant, and it will have the value it had without signal. In general, we could say that when the $z$ component of the
Bloch vector moves far from its original nonsignal value, the limit cycle is perturbed because the signal is too strong, and therefore we are not talking about synchronization anymore. Thus, the parameter that tells us if our system is forced or not is $K \epsilon^{2}$, as some kind of deformation parameter.

Let us consider the case of Fig. 3. Here, $K \epsilon^{2} \approx 0.12$, and therefore $m_{z} \approx 0.88 C$. We observe that the value of $m_{z}$ is close to $C$, but it is not that close. In this regime, the limit cycle is slightly deformed, although we could consider it is still a valid picture for synchronization. In Figs. 4, 5, and 6, the maximum value of the strength is $\epsilon=1$, which corresponds to $K \epsilon^{2}=0.07$. The boundary between synchronization and forced oscillation is not sharply defined, but it seems reasonable to say that we are in the synchronization regime for the parameters values used in these figures.

\section{GENERAL THREE-LEVEL SYSTEM MODEL AND APPEARANCE OF LIMIT CYCLE}

In this section, we contrast the TLS model that we have studied with the three-level model of Refs. [7,17,18] and see how to modify this so that it will have a limit cycle in the same sense as we have described (see Fig. 1). The key difference between these two systems is the nature of the steady state in the absence of signal. In order to explain the fact that the two-level system can be synchronized, we have made use of the interpretation of the mixed state as a probabilistic ensemble of pure states, each of them describing a different and valid limit cycle. In this way, this system is analogous to a classical system that synchronizes in the presence of noise.

In the three-level model studied in Ref. [7], the steady state is the pure state $|0\rangle$ which is an eigenstate of the Hamiltonian. It has a Husimi $Q$ distribution that is equally distributed over all $\phi$, which means that it is a quantum superposition of a set of spin coherent states, each of which are moving along the limit cycle. The crucial difference between our approach and that of Ref. [7] is therefore that we allow statistical mixtures instead of quantum superpositions in the resolution of the stationary state in terms of pure states evolving on a limit cycle. This allows a unified understanding of the synchronization behavior of two- and three-level systems.

In this framework, the stationary state $|0\rangle$ of the Lindblad equation for the three-level model in Ref. [7] is not interpreted as a limit cycle, being one single eigenstate of the Hamiltonian. Only when adding a signal is the stationary state pushed away from this eigenstate and we obtain a cycle. However, in this case it seems natural that there is a substantial deformation of the stationary state, as the limit cycle changes from a single point to a circle.

We can consider a more general three-level system which will show a limit cycle in the same sense as our TLS model. If $\left|m_{z}\right\rangle$ are the eigenstates of the spin-1 operator $S_{z}$, the model of Ref. [7] included transitions only from $|1\rangle$ or $|-1\rangle$ to $|0\rangle$. In the extended model, we allow transitions from $|0\rangle$ to $|1\rangle$ or $|-1\rangle$ in addition.

The modified Lindblad equation in the absence of an external signal would be similar to that of the TLS, although we need to work with a generalized eight-dimensional Bloch sphere if we want to study the motion of the Bloch vector of 
the system [16]:

$$
\frac{d \hat{\rho}}{d t}=\alpha \mathcal{D}\left[\hat{S}_{+}\right] \hat{\rho}+\beta \mathcal{D}\left[\hat{S}_{-}\right] \hat{\rho},
$$

where $\alpha$ and $\beta$ are transition rates. The form of the density matrix for a three-level system is

$$
\hat{\rho}=\left(\begin{array}{ccc}
\frac{1}{\sqrt{3}}+m_{3}+\frac{1}{\sqrt{3}} m_{8} & m_{1}-i m_{2} & m_{4}+i m_{5} \\
m_{1}+i m_{2} & \frac{1}{\sqrt{3}}-m_{3}+\frac{1}{\sqrt{3}} m_{8} & m_{6}-i m_{7} \\
m_{4}+i m_{5} & m_{6}+i m_{7} & \frac{1}{\sqrt{3}}-\frac{2}{\sqrt{3}} m_{8} .
\end{array}\right)
$$

The stationary solution is

$$
\begin{gathered}
m_{1}=m_{2}=m_{4}=m_{5}=m_{6}=m_{7}=0, \\
m_{3}=\frac{-12 \alpha^{2}-6 \alpha \beta}{\sqrt{3}(2 \alpha+4 \beta)(2 \alpha+\beta)}, \\
m_{8}=\frac{1}{2}+\frac{\beta(\alpha-4 \beta)}{2 \alpha(2 \alpha+\beta)} .
\end{gathered}
$$

The Bloch vector that corresponds to the $|0\rangle$ state is $m_{3}=$ $-\frac{\sqrt{3}}{2}, m_{8}=\frac{1}{2}$, and the rest of the components are equal to zero. We see that, in general, the steady state will differ from this one and is instead a mixed state. Thus, in this case we have a mixture of pure states on a circle similar to the one in Fig. 1, which we can see as the analog of the limit cycle in a classical system. Notice that not all values of $\alpha$ and $\beta$ are allowed since we need to keep our states inside an eight-dimensional sphere of radius 1 [16].

\section{CONCLUSIONS}

We have shown that, inside the classical synchronization framework, it is possible to understand that a two-level system provides a valid limit cycle if we interpret mixed states as a probability mixture of pure states with a limit cycle associated to each of them. Explaining the appearance of this cycle was the missing point in previous works $[8,9]$ and is essential for making an analogy with the classical counterpart of synchronization. Equations for the two-level system can be analytically solved and the motion of the Bloch vector in the presence of an external signal can be obtained. Therefore, synchronization can be achieved, but without full phase locking (as is the case for every quantum system that synchronizes, due to quantum noise). Also, the Husimi $Q$ representation is a powerful tool for characterizing the synchronization regimes and strength of phase locking. We also studied the evolution and distortion of the limit cycle with the strength of the signal, since it is fundamental that the signal is too weak to move away from the synchronization regime, as defined classically [1].

With these results for the TLS, we can compare it to the three-level system treated in Refs. $[7,17,18]$. We observe that the response of the system to an external signal is essentially the same for both systems, showing that it is reasonable to consider a qubit to be synchronized in a similar way as a three-level system. Our interpretation of the stationary state as a statistical mixture of pure states following the limit cycle enables a clear interpretation of this behavior. In fact, a more general model for a three-level system than the one used in these previous works is needed if one attempts to understand synchronization starting from a similar limit cycle, since a mixed stationary state is required for following the same argument.

Being able to synchronize such small systems is of great interest because the qubit is the basic unit of quantum computation. Quantum information theory has been in constant development in recent years, and hence learning how these qubits can synchronize can be useful in the quantum computing field. We believe that clarifying the mechanisms by which limit cycles, characteristic of self-sustained oscilltors, arise in quantum systems will help further studies about quantum synchronization.

\section{ACKNOWLEDGMENT}

We thank Jebarathinam Chellasamy for bringing Refs. [5,6] to our attention.
[1] A. Pikovsky, J. Kurths, and M. Rosenblum, Synchronization: A Universal Concept in Nonlinear Sciences, Cambridge Nonlinear Science Vol. 12 (Cambridge University Press, Cambridge, UK, 2001).

[2] B. van der Pol, LXXXVIII: On "relaxation-oscillations," London, Edinburgh, Dublin Philos. Mag. J. Sci. 2, 978 (1926).

[3] T. E. Lee and H. R. Sadeghpour, Quantum Synchronization of Quantum van der Pol Oscillators with Trapped Ions, Phys. Rev. Lett. 111, 234101 (2013).
[4] S. Walter, A. Nunnenkamp, and C. Bruder, Quantum synchronization of two van der Pol oscillators, Ann. Phys. 527, 131 (2015).

[5] G. L. Giorgi, F. Plastina, G. Francica, and R. Zambrini, Spontaneous synchronization and quantum correlation dynamics of open spin systems, Phys. Rev. A 88, 042115 (2013).

[6] V. Ameri, M. Eghbali-Arani, A. Mari, A. Farace, F. Kheirandish, V. Giovannetti, and R. Fazio, Mutual information as an order parameter for quantum synchronization, Phys. Rev. A 91, 012301 (2015). 
[7] A. Roulet and C. Bruder, Synchronizing the Smallest Possible System, Phys. Rev. Lett. 121, 053601 (2018).

[8] I. Goychuk, J. Casado-Pascual, M. Morillo, J. Lehmann, and P. Hänggi, Quantum Stochastic Synchronization, Phys. Rev. Lett. 97, 210601 (2006).

[9] O. V. Zhirov and D. L. Shepelyansky, Synchronization and Bistability of a Qubit Coupled to a Driven Dissipative Oscillator, Phys. Rev. Lett. 100, 014101 (2008).

[10] H. Eneriz, D. Z. Rossatto, F. A. Cárdenas-López, E. Solano, and M. Sanz, Degree of quantumness in quantum synchronization, Sci. Rep. 9, 19933 (2019).

[11] G. L. Giorgi, F. Galve, G. Manzano, P. Colet, and R. Zambrini, Quantum correlations and mutual synchronization, Phys. Rev. A 85, 052101 (2012).

[12] G. Manzano, F. Galve, and R. Zambrini, Avoiding dissipation in a system of three quantum harmonic oscillators, Phys. Rev. A 87, 032114 (2013).
[13] G. Manzano, F. Galve, G. L. Giorgi, E. Hernández-García, and R. Zambrini, Synchronization, quantum correlations, and entanglement in oscillator networks, Sci. Rep. 3, 1439 (2013).

[14] M. Le Bellac and P. de Forcrand-Millard, Quantum Physics (Cambridge University Press, Cambridge, UK, 2006).

[15] R. Gilmore, C. M. Bowden, and L. M. Narducci, Classicalquantum correspondence for multilevel systems, Phys. Rev. A 12, 1019 (1975).

[16] S. K. Goyal, B. Neethi Simon, R. Singh, and S. Simon, Geometry of the generalized Bloch sphere for qutrits, J. Phys. A: Math. Gen. 49, 165203 (2016).

[17] A. Roulet and C. Bruder, Quantum Synchronization and Entanglement Generation, Phys. Rev. Lett. 121, 063601 (2018).

[18] M. Koppenhöfer and A. Roulet, Optimal synchronization deep in the quantum regime: Resource and fundamental limit, Phys. Rev. A 99, 043804 (2019). 